\title{
Une langue de théâtre composée d'accents multiples
}

A Theatre Language Composed of Multiple Accents

Béatrice Picon-Vallin

\section{(2) OpenEdition}

Journals

Édition électronique

URL : https://journals.openedition.org/rsl/2613

DOI : $10.4000 /$ rsl.2613

ISSN : 2271-6246

Éditeur

Éditions Rue d'Ulm

Référence électronique

Béatrice Picon-Vallin, "Une langue de théâtre composée d'accents multiples », Revue Sciences/Lettres [En ligne], 6| 2019, mis en ligne le 10 décembre 2018, consulté le 30 juillet 2021. URL : http:// journals.openedition.org/rsl/2613; DOI : https://doi.org/10.4000/rsl.2613

Ce document a été généré automatiquement le 30 juillet 2021.

(C) Revue Sciences/Lettres 


\title{
Une langue de théâtre composée d'accents multiples
}

\author{
A Theatre Language Composed of Multiple Accents
}

Béatrice Picon-Vallin

1 C'est petit à petit que la troupe du Théâtre du Soleil, créée en 1964 et installée en 1970 à la Cartoucherie de Vincennes, s'enrichit d'étrangers ${ }^{1}$. Leur présence n'est ni une déclaration de principe, ni un manifeste, elle est une évidence : on vient, et si on reste, on travaille, on joue. Et si on a un accent, on le travaille, on l'adoucit ou on le garde, à condition d'être compréhensible - le public étant cependant aidé par le jeu physique. Par la suite, dans les stages qui vont s'organiser pour renouveler la troupe, Ariane Mnouchkine retiendra des comédiens en fonction de leur personne, de leurs potentialités de comédiens, non en fonction du degré de connaissance du français. Et au fur et à mesure des arrivées, un membre de la troupe se consacrera à faire répéter spécialement ceux qui ont des difficultés avec le texte. Mais les choses se passent organiquement, sans plan préconçu.

Différent est le cas de la troupe de Peter Brook à Paris : en 1971, il fonde le Centre international de recherche théâtrale (CIRT), qui devient, lors de l'ouverture des Bouffes du Nord, le Centre international de créations théâtrales (CICT). «International » est posé dans la dénomination du groupe formé et on se souvient du Mahabharata (1985) où des artistes européens, africains et asiatiques dialoguaient en français pour mettre en scène l'histoire de l'humanité que représente ce long poème indien (Andrzej Seweryn, Ryszard Cieslak, Bruce Myers, Vittorio Mezzogiorno, Sotigui Kouyaté et tant d'autres). La version filmée sera en anglais et par la suite les derniers spectacles de Peter Brook seront joués par des acteurs parlant anglais. Les démarches divergent, mais ce sont bien là les deux grands lieux où dans les années 1970 la langue théâtrale française se métisse et fait chanter nos mots, nos phrases, au point qu'on les entend autrement, plus fort - comme venant d'ailleurs ${ }^{2}$. De cet ailleurs qu'est le théâtre dans sa toute-présence même.

3 La question est essentielle et le risque est donc grand de ne faire que l'effleurer. Tentons une première approche. Aux débuts du Théâtre du Soleil donc peu de ses 
membres ont «des accents » - celui de Roberto Moscoso, arrivé en France à seize ans, est léger et " élégant, fin ». Car parfois, même avec une langue parfaite, il reste un je ne sais quoi qui rend la langue plus belle, laisse filtrer une musique autre que celle du français parlé dans l'île-de-France. Il est difficile de dresser les listes des entrées au Soleil, mais dès Les Clowns (1968), la troupe compte un Fabrice Herrero, déjà là pour Le Songe d'une nuit d'été (1968), Argentin à l'accent très marqué, qui pratique le plateau, mais s'occupe surtout des maquillages et des accessoires, et un Mario Gonzalès, comédien qui vient du Guatemala. On entendra leur façon de dire le français dans 1789 ou La révolution doit s'arrêter à la perfection du bonheur (1970), ainsi que celle de Myrrha Donzenac, d'origine martiniquaise, qui participe à la séquence du grand récit de la Révolution fait par les bateleurs sur leurs tréteaux, s'adressant directement au peuple de Paris de 1970 debout devant eux, lui parlant à l'oreille, en allant du cri au chuchotis [Lien au dossier pédagogique multimédia en ligne « Entendre le théâtre », à venir début juillet 2019]. Arrivés pour 1793, trois Marseillais à l'accent du Sud plus ou moins prononcé participent à la reprise de 1789 et aux accents étrangers s'ajouteront les accents locaux.

Sophie Moscoso, alors assistante d'Ariane Mnouchkine, témoigne :

Lorsque j'ai connu le Soleil, puis que j'y ai travaillé dans les années 70, je n'ai jamais pris garde aux accents. Il faut dire qu'ils étaient peu nombreux, à l'époque. Et ne gênaient pas le travail théâtral. Sur le plateau, ils ne posaient pas particulièrement de problèmes de compréhension. Lorsque les Marseillais sont arrivés, Ariane essayait parfois d'atténuer celui de Maxime Lombard qui était le plus remarquable ; celui de Jean-Claude Bourbault était le moins prononcé3.

5 Pour 1793, ils jouaient tous trois des Marseillais montés à la capitale et leur accent crédibilisait leurs personnages. Dans L'Âge d'or, Philippe Caubère jouait Abdallah, immigré arabe qui débarque en France. "Avec un masque, qui plus est », commente Sophie Moscoso. «Le Marseillais a adopté comme comédien l'accent d'un immigré arabe. » Cet accent du Sud perdure, avec des variantes, jusqu'à aujourd'hui dans Une chambre en Inde avec Maurice Durozier, originaire de Perpignan.

6 Mais le back-stage lui aussi accueille les étrangers; la Babel qui commence à se construire au Théâtre du Soleil n'est pas seulement sur le plateau mais à la technique, aux masques, à la couture, à l'administration, aux cuisines, partout, et il me semble que c'est aussi de cela qu'il faut parler : un micromonde à l'image du grand monde qui s'ouvre entre progressivement au Soleil, et concrètement dans cette grande maison qu'est la Cartoucherie; chacun y trouve une façon de travailler au mieux, et de s'adresser aux autres et au public, à travers cette ambiance sonore générale très particulière. Ehrard Stiefel, le facteur de masques et son délicat accent suisse, est là depuis Le Songe d'une nuit d'été; Jonathan Sutton arrive pour L'Âge d'or, et son accent anglais (précisons: «dit anglais », car il y en a tant au Royaume-Uni...) disparaît presque sous le jeu masqué; Hector Ortiz vient de la République dominicaine où il a été torturé - «Hector, le gardien de la Cartoucherie, toujours souriant, avec un accent chantant, vraiment $»^{4}$. Liliana Andreone, réfugiée argentine, arrive en 1976 au Soleil avec le film Molière ou la vie d'un honnête homme; elle ne parle pas encore français ou très peu, l'apprend grâce à l'organisme de la Cimade. C'est d'ailleurs ainsi que le Théâtre du Soleil sera connecté avec la Cimade et que plus tard Hélène Cinque organisera des stages de théâtre pour cette association dans la salle de répétitions du Soleil pour permettre aux réfugiés d'Amérique latine de mieux présenter leur dossier, de mieux défendre leurs droits ${ }^{5}$. Juste circulation des choses entre les langues et le théâtre. Avec 
Liliana, qui est d'abord à la couture, deux autres Argentins sont là et travaillent sur les décors de Molière. En plus de l'accent espagnol des réfugiés politiques, c'est aussi le portugais qui retentit avec une bande de réfugiés économiques cette fois, qui forment l'équipe technique des "Portugais», comme on les appelle - Antonio Ferreira (pour 1793), puis Victor Costa, José Vasconcelos, Joachim Baptista. Ils travaillent avec GuyClaude François. « Entre eux, ils parlaient portugais et ont gardé leurs accents plus ou moins prononcés ${ }^{6} . »$ Liliana Andreone, qui va ensuite et jusqu'à aujourd'hui travailler aux relations personnalisées avec les comités d'entreprise ou les spectateurs qui caractérisent le Soleil, souligne que son accent lui a ouvert des portes, ses interlocuteurs étant souvent curieux d'en savoir plus sur les évènements qui se passaient en Amérique latine, particulièrement dans les années 1970. Elle rit, commente : «Les accents ouvraient la tête », et elle ajoute : « Ils mettent l'être humain dans un contexte plus grand », proposant ici une belle définition du lieu théâtral.

7 L'acteur Duccio Bellugi-Vannuccini raconte qu'assistant en tant que spectateur à L'Histoire terrible mais inachevée de Norodom Sihanouk, roi du Cambodge (1985), il ne s'est pas aperçu de la présence de ces accents : pour lui, d'origine italienne, on y parlait un français étrange, mais lié à la narration théâtrale ; pourtant, ils étaient déjà nombreux, sur le plateau et en dehors, arrivés, avec les comédiens, du Chili, du Brésil. Un jeune Khmer, Ly Nissay, qu'Ariane Mnouchkine et Hélène Cixous avaient rencontré, lors de leur voyage pour préparer ce spectacle, dans un camp à la frontière de la Thaïlande et du Cambodge où il avait fui la terreur des Khmers Rouges, et qu'elles avaient fait venir en France avec sa famille, était aussi à la Cartoucherie, nouveau revenu de loin, fabriquant, avec d'autres, les statuettes qui représentaient son peuple sur la scène du Soleil. C'est plus tard et dans la pratique que Duccio Bellugi-Vannuccini a pris conscience du grand chant du monde: d'abord dans l'éblouissement d'un stage organisé en 1986 au Soleil où les participants venaient de tous les coins du monde ; puis dans le travail suivant, L'Indiade ou l'Inde de leurs rêves (1987), où de nouveaux comédiens sont arrivés d'Iran, de Tunisie, d'Algérie, d'Inde, du Chili, du Brésil...

8 Tous les accents étaient acceptés s'ils étaient retravaillés. Ainsi Maurice Durozier raconte comment Ariane Mnouchkine lui faisait au début écouter des enregistrements de la chanteuse Damia. «Le but du travail, précise Duccio Bellugi-Vannuccini, n'était pas de ne plus avoir d'accent, de le supprimer, mais de ne pas faire reconnaître immédiatement son origine ${ }^{7}$. » Ainsi pour Le Tartuffe (1995), Shahrokh Meshkin Ghalam qui jouait le rôle-titre ne devait pas être identifié comme Iranien, il était quelqu'un venu de cet ailleurs d'Asie de l'Ouest, mais non typique, non réductible à un pays. C'est d'ailleurs ici une autre différence avec le travail théâtral de Peter Brook qui, comme en témoigne Liliana Andreone qui a collaboré à plusieurs reprises avec sa troupe au Festival de Berlin, considérait que, si le comédien était dans le rôle, les spectateurs n'entendaient pas son accent, alors qu'Ariane Mnouchkine faisait travailler le texte pour que l'origine d'un comédien ne puisse pas influencer le personnage, la position du spectateur sur le personnage ${ }^{8}$.

9 Duccio Bellugi-Vannuccini a travaillé avec différentes personnes - plus personnellement avec Georges Roiron, qui insistait sur la prononciation, la diction, la phonétique. Et, comme tant d'autres, avec Françoise Berge, entrée au Soleil en 1981 et toujours là, dans une fonction qu'elle assume en étroite relation avec Ariane Mnouchkine et les demandes que celle-ci formule pour les spectacles et les comédiens ${ }^{9}$. 
Il se souvient aussi du travail très difficile sur Le Tartuffe (1995) pour l'apprentissage du e muet français et des liaisons dans l'alexandrin.

Mais de toutes façons «quand tu parles dans une autre langue que la tienne, chaque mot prend une importance particulière, cela enlève le réalisme quotidien ", souligne-t$\mathrm{il}^{10}$, et c'est sans doute un des moyens qu'a saisis Ariane Mnouchkine pour arriver à ce parler théâtral qu'elle revendique, et qui n'est pas une langue normée. Ni supprimer l'accent, ni l'exacerber. Être compréhensible. La question se pose de l'harmonie à trouver entre ces différents accents : le travail préparatoire de Françoise Berge joue ici un rôle important (ainsi, pour Le Tartuffe, il fallait trouver la cohérence d'une famille réunissant des comédiens de pays différents). Et la musique créée et jouée par JeanJacques Lemêtre ${ }^{11}$, présent à toutes les répétitions, à l'écoute des comédiens, et avec qui Françoise Berge est en totale entente, tend à fluidifier, unifier sans effacer la variété.

11 Françoise Berge, qui a d'abord été prospectrice au Festival mondial de théâtre de Nancy, a eu très tôt l'habitude d'entendre des spectacles en langue étrangère et sans sur-titrage. Venant de la linguistique et de la psychanalyse, elle fait travailler les acteurs qu'elle prend à leurs différents niveaux de connaissance du français, et souvent les fait partir de «l'enfance de la langue »: des comptines par exemple, qui ont pour but de fixer la langue maternelle et permettent d'« ingérer » la nouvelle langue. Elle distingue des «langues ouvrantes » qui permettent d'entendre les sonorités des autres langues (langues de la plupart des pays de l'Est par exemple), et d'autres, comme l'italien et l'anglais, « qui ont un éventail d'écoute rétréci ». Le travail sur les accents se fait à partir de l'écoute, et en cela ressemble au travail du jeu de l'acteur dont c'est la base. Elle parle des efforts énormes que les comédiens ont à fournir pour arriver à ce parler théâtral qui porte le sens avec ou sans accent, et cite en exemple l'expérience du comédien chilien Andrés Pérez Araya qui jouait le rôle de Gandhi dans L'Indiade (1987) et dont Ariane Mnouchkine voulait qu'il "féminise sa voix ", adoucisse les tonalités " macho-latinos » et les espagnolismes chiliens trop forts ${ }^{12}$. Pourtant, pour jouer Zhou Enlai dans L'histoire terrible mais inachevée de Norodom Sihanouk, roi du Cambodge (1985), "Andrés Pérez avait transformé son handicap quant à la difficile maîtrise du français en atout créatif, son accent chilien se prêtant alors curieusement à merveille à l'idée d'un accent chinois. Il n'y a pas vraiment eu d'effort pour cela toutefois, l'impression étant là d'emblée, car Andrés passait par une transformation physique qui faisait de lui un sosie de son personnage ${ }^{13}$."

12 Il est important de réfléchir à la façon dont certains comédiens peuvent, en scène, perdre totalement leur accent - qui peut se caractériser par les intonations, les inflexions, l'intensité de la voix placée sur telle ou telle syllabe, la façon de prononcer le $\mathrm{r}$ ou le l, de mouiller certaines voyelles, selon la langue d'origine. Ainsi Marilu Marini, venue d'Argentine elle aussi, et qui dans la vie parlait avec un accent espagnol puissant et rocailleux, le fait disparaitre ou l'oublie totalement pour le rôle de la Reine dans Molière : travail du corps sans doute, entraînement de la voix, ou énergie particulière distillée par le plateau? Certains étrangers au Soleil n'ont aucun accent. D'autres n'arrivent pas à le perdre, certains encore, peu nombreux, ont du mal à parler français, malgré le travail qu'ils font au Soleil sur la langue. L'acquisition totale du français, la perte ou non de l'accent sont sans doute liés aux circonstances, au vécu des personnes, réfugiés politiques plus ou moins orphelins de leur langue et de leur pays, au contexte de leur départ, ou de leur exil, aux traumatismes vécus lors de cette séparation de la langue maternelle et au degré de volonté de survie. Chaque cas est différent. 
13 Duccio Bellugi-Vannuccini, qui a gardé un léger accent, cherche pour chacun de ses personnages une origine étrangère - italienne, allemande, pas française ; en scène il se donne, ou plutôt il leur donne une origine étrangère, « mais au fond, dit-il, je n'ai plus de langue originelle ; l'italien est devenu une langue étrangère pour moi, j'ai en italien un accent bâtard ${ }^{14}$ ». La langue de théâtre passe à travers le corps bien sûr, surtout pour lui qui de plus vient du mime et de la danse. Mais aujourd'hui il peut travailler des accents différents car, à travers toutes ces sonorités entendues quotidiennement au Théâtre du Soleil et le travail vocal accompli sur chaque rôle, son oreille s'est particulièrement développée.

14 À partir des années 1980, les étrangers sont devenus nombreux dans la troupe : « Après une vague d'Italiens, il y a eu une vague de réfugiés sud-américains, une grosse vague brésilienne, puis une énorme vague afghane », dit encore Duccio Bellugi-Vannuccini. Et aujourd'hui il y a vingt-cinq nationalités dans Une chambre en Inde. Une règle a été posée : tous doivent parler français entre eux, c'est devenu une obligation, pour que la communication entre les comédiens, les stagiaires, les ateliers, dans la vie quotidienne de travail, soit facilitée et qu'un training soit à l'œuvre pour l'apprentissage ou le perfectionnement de la langue française, celle du pays où vit la troupe et celle du plateau.

15 Mais c'est une richesse infinie que ces accents que les différentes langues posent sur la langue de la troupe; même ceux qui n'ont pas d'accent, mais viennent de cultures différentes - comme Simon Abkarian, chez qui plusieurs couches culturelles se sont empilées et comme combinées (Liban, diaspora arménienne, États-Unis) -, ont une manière de dire le français, d'en changer légèrement la prosodie, qui étrangéise le parler par l'attaque de la phrase, le rythme, une intensité parfois accrue sur telle ou telle syllabe. D’autres émeuvent par le léger ralentissement du débit, ou une petite difficulté d'énonciation.

16 Avec quelques années de plus, l'utopie de départ du Théâtre du Soleil, « faire le plus beau théatre du monde ", s'est chargée d'une autre signification : cela voulait dire aussi prendre à bras le corps les problèmes du monde, faire entrer le monde dans la troupe, et en s'élargissant au monde, faire parler théâtralement les uns et les autres, voisins et lointains camarades, éventuellement faire entendre des témoins.

17 La question des accents sur scène va donc devenir celle des langues. La possibilité de projeter facilement la traduction sur un écran ou bien n'importe où dans l'espace scénique a permis la retransmission directe de témoignages enregistrés et diffusés en Vo, accompagnée d'une traduction simultanée calligraphiée dans Le Dernier Caravansérail (2003), où le public était profondément touché par les sonorités authentiques des langues des pays-martyrs. À cette possibilité exploitée avec efficacité s'ajoute l'apprentissage, le partage des langues des uns et des autres, dans des répliques échangées et traduites en direct par des sur-titrages sur diverses parties du dispositif scénique. On retrouve bien la question des accents, mais c'est un accent étranger aux langues qui sont parlées (l'anglais par une Française par exemple). Dans les créations collectives des années 2000, ce n'est plus seulement la question des accents rendue plus complexe, mise en abyme, mais aussi celle des langues et de leur traduction qui vivifient la scène.

18 Une chambre en Inde (2016) fonctionne totalement sur ce principe : outre leurs langues maternelles parlées par les comédiens, on entend le russe parlé par un Arménien, par une Française, les différentes façons de parler anglais des Français, dont celle du Sud- 
Ouest, celles des Pakistanais, des Afghans, et bien d'autres, le japonais d'une comédienne française qui répond au japonais de deux Japonais, etc. Évoquons rapidement trois autres cas qui constituent des dates dans l'histoire du thêâtre.

D'abord, la traduction en langue khmer, langue des évènements racontés dans L'Histoire terrible mais inachevée de Norodom Sihanouk, roi du Cambodge, pièce d'Hélène Cixous. La prise en charge du texte et de sa mise en scène originelle, sa recréation au Cambodge par une troupe khmer, accompagnée par deux acteurs du Soleil - Georges Bigot qui avait joué Sihanouk et Delphine Cottu -, et sa venue sur le plateau même du Théâtre du Soleil ont été un évènement à la fois politique et émotionnel. Entendre les sonorités de la langue khmer en lisant la traduction, qui était en fait un original, redoublait la force du propos et l'intensité de l'écoute du texte : il devenait comme sonorisé (2013).

Deuxième exemple : l'apprentissage du tamoul par tous les acteurs du Soleil dans un long atelier conduit par P.K. Sambandan, un maître de terukkuttu (forme théâtrale très ancienne du Sud de l'Inde jouée dans les campagnes), accompagné d'une comédienne qui possédait la langue (Nirupama Nityanandan, tamoulophone et anglophone). Cet atelier difficile, où il fallait mémoriser texte, danse, musique et chant, a abouti à l'introduction de deux séquences du Mahabharata, en terukkuttu, jouées par les acteurs du Soleil dans Une chambre en Inde. Ajoutons qu'en 1997 déjà, pour Et soudain des nuits d'éveil, Duccio Bellugi-Vannuccini avait appris à parler en tibétain pour dire une prière après sa fameuse "Danse du cerf», et toute une conférence de presse, traduite consécutivement en français par Sergio Canto, Mexicain. Au Soleil, les processus sont organiques et se développent sur la durée.

21 Enfin, dans les soirées du Mahabharata (Le Mahabharata. Hommage à notre maître..., 2018), les élèves tamouls de P.K. Sambandan, venus d'Inde, collaboraient avec la troupe du Soleil pour quatre épisodes. On ne pouvait pas vraiment distinguer qui étaient les acteurs du Soleil (vingt-huit nationalités redisons-le) et qui étaient les Indiens. Nul doute que la langue tamoule se transformait dans le gosier et les cordes vocales des acteurs de la troupe française multinationale, mais qui pouvait s'en rendre compte en France ? Peut-être certains spectateurs, car ne l'oublions pas, dans le foyer du Soleil où le public est international, on entend aussi et depuis longtemps langues et accents différents. Et puis ajoutons ce dernier exemple : jouée au Théâtre du Soleil en 2018, la Trilogie de Simon Abkarian y a fait à son tour fleurir tout un bouquet d'accents qui évoque la diversité des pays de la Méditerranée sans qu'on puisse en désigner un en particulier. Cette Trilogie est le fruit de l'expérience du Soleil.

Il s'agit sans doute ici d'un travail, aux facettes et approches multiples, de compréhension du jeu des écarts - non des différences - comme dirait François Jullien, entre les langues et les accents, d'un travail sur le commun du monde pour le partager en scène et hors scène dans la Maison-Soleil : accents, langues, traductions, ce sont toutes les étapes par lesquelles passent les acteurs, les équipes qui les soutiennent à tous les postes nécessaires et les spectateurs qui à la Cartoucherie font aussi scintiller la langue française par leurs prononciations, inflexions et intonations.

Terminons ce début de réflexion par ces lignes d'Hélène Cixous qui élargissent un peu la problématique, mais où l'on comprend que cette troupe aux accents variés qui ouvre l'espace scénique à un langage nouveau et toujours renouvelé, conférant une étrange poésie à la langue française, est composée d'artistes-voyageurs créant aujourd'hui comme au carrefour des langues. 
Il existe une Transespèce humaine, ou plutôt humanimale, une population composée d'êtres qui sont de nature hospitalière, des vivants d'une étoffe que je trouve merveilleuse, toujours encore en tissage et en métissage. Leur nature échappe aux définitions territoriales, nationales, identitaires. S'ils ont pris leur source dans différentes clôtures, géopolitiques, s'ils sont "nés » afghans, chinois, miq maq, français, togolais, norvégiens, mapuches, féroïens, khmers, uruguayens, éthiopiens (à suivre...), ils ont par la suite transporté leur cours à travers pays et continents. En rencontrant bien d'autres et frottant leurs cervelles à ta cervelle, en s'exposant toujours, joyeusement, à bien d'autres, ouverts au risque de la surprise, ils sont ouverts, larges, et toujours en métamorphose, passant d'un âge à l'autre sexe, octogénaires de trente ans, génies curieux, aventuriers des temps, résistant dans la pratique aux tentations paresseuses de l'Appartenance et du Propre.

Ce ne sont pas des fantômes, ni des habitants des rêves. Ils ont des papiers. Ils obtiennent des visas. Mais naturellement, ils ne se prennent pas pour leurs papiers. Plutôt pour des poèmes, et toujours en traduction. Ils écoutent, ils ont l'oreille gourmande et la langue enchantée ${ }^{15}$. Ces amis de l'amour plutôt que de la haine, vous les aurez reconnus, n'est-ce pas ? Ce sont les Acteurs ${ }^{16}$.

\section{NOTES}

1. Même si les ascendances de certains sont russe, anglaise ou italienne.

2. On peut signaler Les Bas-Fonds, monté au Théâtre de la Ville par Lucian Pintilié en 1983 (la date aurait dû être 1981, mais a été reculée à cause d'un incendie au Théâtre) dont la distribution était très internationale avec Bruce Myers, Yaruzer Centikaya, Dragan Nicolic, entre autres.

3. Entretiens réalisés à Paris en juillet et septembre 2018.

4. Ibid.

5. Entretien réalisé à Paris, le 22 octobre 2018.

6. Voir note 3.

7. Entretien réalisé à Paris, le 21 octobre 2018.

8. Voir note 5.

9. Sa fonction de « coach » est ainsi notée dans les premiers génériques : «Travail de phonétique et de linguistique » pour L'Indiade, "Travail de phonétique et de diction » pour La Ville parjure. Mais son travail est plus complexe. Voir infra.

10. Voir note 7.

11. Le cas du Tartuffe excepté, car il n'y avait qu'une bande sonore pour la pièce de Molière, déjà musicale par ses vers.

12. Entretien réalisé à Paris, le 3 novembre 2018.

13. Entretien avec Jean-François Dusigne, Paris, le 9 février 2019.

14. Voir note 7.

15. C'est nous qui soulignons...

16. Hélène Cixous, citée par Ariane Mnouchkine dans l'« Éditorial du Théâtre du Soleil » consacré à Kanata : « Histoire d'une admiration ». https://www.theatre-du-soleil.fr/fr/les-editos/editorialdu-22-octobre-2018-28 


\section{RÉSUMÉS}

Alors que la troupe de Peter Brook, le Centre international de recherche théâtrale, fondé en 1971 à Paris, affiche dans son intitulé son caractère «international ", c'est petit à petit que la troupe du Théâtre du Soleil, créée en 1964 et installée en 1970 à la Cartoucherie de Vincennes, s'enrichit d'étrangers. Leur présence n'est ni une déclaration de principe, ni un manifeste, mais une réalité. Les accents sont travaillés, adoucis ou gardés, à condition d'être compréhensibles. Avec des démarches divergentes, ce sont là les deux grands lieux où, dans cette période, la langue théâtrale française se métisse et fait chanter ses mots au point qu'on les entend autrement, plus fort, comme venant d'ailleurs. La question est essentielle et mériterait une étude approfondie. Il s'agit, dans cette contribution, de tenter une première approche de la déjà très longue histoire de l'inscription des accents, régionaux ou lointains, dans le parler théâtral original du Théâtre du Soleil, histoire enrichie dans un second temps par celle de l'inscription des langues et de leurs traductions, qui croise la première sans la recouvrir. Une approche qui ne concerne pas seulement les acteurs, mais les équipes qui les soutiennent à tous les postes nécessaires, et les spectateurs de la Cartoucherie.

Whereas Peter Brook's theatre company, the International Centre for Theatre Research, founded in 1971 in Paris, displays its "international" character in its title, the Théâtre du Soleil, created in 1964 and installed in 1970 at the Cartoucherie de Vincennes, welcomed foreigners little by little. Their presence was neither a statement of principle nor a manifesto, but a reality. Accents were worked on, softened or kept as long as they could be understood. These theatre companies with their different approaches were the two major venues where, during this period, French theatrical language involved a mix, making its words sing out to the extent where people heard them differently, more strongly, as coming from elsewhere. This issue is essential and merits an in-depth study. The present article is a first approach to the history, already quite lengthy, of the inclusion of accents, regional or non-native, into the original theatrical speech of the Théatre du Soleil, a history further enriched by the inclusion of languages and their translations, which intersects with the former without encompassing it. This approach concerns the actors, the teams that support them in all needed positions and the spectators at the Cartoucherie.

\section{INDEX}

Keywords : Théâtre du Soleil, Ariane Mnouchkine, Peter Brook, language, native language, translation, multinational theatre company, theatrical speech, foreign accents, local accents Mots-clés : Théâtre du Soleil, Ariane Mnouchkine, Peter Brook, langue, langue maternelle, traduction, troupe multinationale, parler théâtral, accents étrangers, accents locaux

\section{AUTEUR}

\section{BÉATRICE PICON-VALLIN}

Béatrice Picon-Vallin est directrice de recherches émérite au CNRS (THALIM). Elle a dirigé pendant neuf ans le Laboratoire de recherches sur les arts du spectacle (LARAS). Elle dirige trois collections (« Arts du spectacle », CNRS Éditions ; «thXX », L’Âge d'Homme ; « Mettre en scène », Actes Sud-Papiers). Elle est l'auteur de nombreux ouvrages, en particulier Meyerhold, Les voies de la création théâtrale, vol. 17, CNRS Éditions 
(1990-1999-2004, plusieurs traductions), ainsi que les Écrits sur le théâtre de V. Meyerhold (4 volumes). Elle a dirigé des ouvrages collectifs (Le Film de théâtre; La Taganka. Lioubimov; Meyerhold. La mise en scène dans le siècle/ Mejerhold. Rezissura $v$ perspektive veka; La Scène et les images), fruits de programmes collectifs du CNRS ou de collaborations internationales. Elle a aussi publié de nombreux articles et études sur le théâtre en URSS/Russie au XX ${ }^{\mathrm{e}}-\mathrm{XXI}^{\mathrm{e}}$ siècle, en particulier sur les avant-gardes des années 1920, sur la mise en scène et le jeu de l'acteur, sur le théâtre et les autres arts (musique, cirque, cinéma, vidéo...), et sur les relations entre la scène et les nouvelles technologies. Elle a enseigné pendant dix ans l'histoire du théâtre au CNSAD de Paris, intervient actuellement dans différentes écoles de théâtre en France et à l'étranger.

Parmi ses publications :

Ariane Mnouchkine, Arles, Actes Sud-Papiers, 2009 (plusieurs traductions).

V. Meyerhold, Écrits sur le théâtre, B. Picon-Vallin (éd.), nouvelle édition revue et augmentée, vol. 2, Lausanne, L’Âge d'Homme, 2009 ; vol. 3 sous presse.

Le Théâtre du Soleil. Les cinquante premières années, Arles, Actes Sud, 2014 (trad. au Brésil, Éditions Perspectiva ; traduction aux États-Unis en cours chez Routledge).

Meyerhold, Le Cocu magnifique, B. Picon-Vallin (éd.), Valenciennes, Éditions Subjectile, « Rewind », 2018, livre numérique.

Les Théâtres documentaires, B. Picon-Vallin et E. Magris (dir.), Montpellier, Éditions

Deuxième Époque, 2019. 